\title{
CALCULATION OF CHAKALOV-POPOVICIU QUADRATURES OF RADAU AND LOBATTO TYPE
}

\author{
MIODRAG M. SPALEVIĆ ${ }^{1}$
}

(Received 25 January, 1999; revised 19 April, 2001)

\begin{abstract}
A numerical method for calculation of the generalized Chakalov-Popoviciu quadrature formulae of Radau and Lobatto type, using the results given for the generalized ChakalovPopoviciu quadrature formula, is given. Numerical results are included. As an application we discuss the problem of approximating a function $f$ on the finite interval $I=[a, b]$ by a spline function of degree $m$ and variable defects $d_{\nu}$, with $n$ (variable) knots, matching as many of the initial moments of $f$ as possible. An analytic formula for the coefficients in the generalized Chakalov-Popoviciu quadrature formula is given.
\end{abstract}

\section{Introduction}

Let $d \lambda(t)$ be a nonnegative measure on the real line $\mathbb{R}$, with compact or infinite support $\operatorname{supp}(d \lambda)$, for which all moments

$$
\mu_{k}=\int_{\mathbb{R}} t^{k} d \lambda(t), \quad k=0,1, \ldots,
$$

exist and are finite, and $\mu_{0}>0$. A quadrature formula of the form

$$
\int_{\mathbb{R}} f(t) d \lambda(t)=\sum_{\nu=1}^{n} \sum_{i=0}^{2 s} A_{i, \nu} f^{(i)}\left(\tau_{\nu}\right)+R(f),
$$

where $A_{i, \nu}=A_{i, v}^{G}=A_{i, \nu}^{(n, s)}, \tau_{v}=\tau_{\nu}^{(n, s)}$, which is exact for all algebraic polynomials of degree at most $2(s+1) n-1$, was considered firstly by P. Turán (see [20]), in the case when $d \lambda(t)=d t$ on $[-1,1]$. The case with a weight function, $d \lambda(t)=\omega(t) d t$ on the interval $[a, b]$, has been considered by the Italian mathematicians Ossicini, Ghizzetti,

\footnotetext{
${ }^{1}$ University of Kragujevac, Faculty of Science, Department of Mathematics and Informatics, P.O. Box 60, 34000 Kragujevac, Serbia, Yugoslavia; e-mail: spale@knez.uis.kg.ac.yu.

(C) Australian Mathematical Society 2002, Serial-fee code 0334-2700/02
} 
Guerra and Rosati, and also by Chakalov, Stroud, Stancu, Ionescu, Pavel, etc. (see [15] for references).

The nodes $\tau_{v}$ in (1.1) must be zeros of a (monic) polynomial $\pi_{n}(t)$ which minimizes the integral

$$
F \equiv F\left(a_{0}, a_{1}, \ldots, a_{n-1}\right)=\int_{\mathbb{R}} \pi_{n}(t)^{2 s+2} d \lambda(t)
$$

where

$$
\pi_{n}(t)=t^{n}+a_{n-1} t^{n-1}+\cdots+a_{1} t+a_{0}
$$

In order to minimize $F$ we must have

$$
\int_{\mathbb{R}} \pi_{n}(t)^{2 s+1} t^{k} d \lambda(t)=0, \quad k=0,1, \ldots, n-1 .
$$

Polynomials $\pi_{n}(t)$ which satisfy this new type of orthogonality "power orthogonality" are known as $s$-orthogonal (or $s$-self associated) polynomials with respect to the measure $d \lambda(t)$.

For $s=0$ we have the standard case of orthogonal polynomials.

Let $n \in N$ and let $\sigma=\sigma_{n}=\left(s_{1}, s_{2}, \ldots, s_{n}\right)$ be a sequence of nonnegative integers. A generalization of the Gauss-Turán quadrature formula (1.1) to rules having nodes with arbitrary multiplicities was given, independently, by Chakalov $[2,3]$ and Popoviciu [17].

In this case, it is important to assume that the nodes $\tau_{v}\left(=\tau_{v}^{(n, \sigma)}\right)$ are ordered, say

$$
\tau_{1}<\tau_{2}<\cdots<\tau_{n}, \quad \tau_{v} \in \operatorname{supp}(d \lambda),
$$

with odd multiplicities

$$
2 s_{1}+1,2 s_{2}+1, \ldots, 2 s_{n}+1
$$

respectively. Then the corresponding quadrature formula

$$
\int_{\mathbb{R}} f(t) d \lambda(t)=\sum_{\nu=1}^{n} \sum_{i=0}^{2 s_{\nu}} A_{i, \nu} f^{(i)}\left(\tau_{\nu}\right)+R(f),
$$

where $A_{i, \nu}=A_{i, \nu}^{G}=A_{i, \nu}^{(n, \sigma)}, \tau_{\nu}=\tau_{\nu}^{(n, \sigma)}$, has the maximum degree of exactness

$$
d_{\max }=2 \sum_{\nu=1}^{n} s_{\nu}+2 n-1
$$

if and only if

$$
\int_{\mathbb{R}} \prod_{v=1}^{n}\left(t-\tau_{v}\right)^{2 s_{i}+1} t^{k} d \lambda(t)=0, \quad k=0, \ldots, n-1
$$


The last orthogonality conditions correspond to (1.2). The existence of such quadrature rules has been proved by Chakalov [2], Popoviciu [17] and Morelli and Verna [16] and existence and uniqueness subject to (1.3) by Ghizzetti and Ossicini [10].

The conditions (1.6) define a sequence of polynomials $\left\{\pi_{n, \sigma}\right\}_{n \in N_{0}}$,

$$
\pi_{n, \sigma}(t)=\prod_{\nu=1}^{n}\left(t-\tau_{\nu}^{(n, \sigma)}\right), \quad \tau_{1}^{(n, \sigma)}<\tau_{2}^{(n, \sigma)}<\cdots<\tau_{n}^{(n, \sigma)}, \tau_{\nu}^{(n, \sigma)} \in \operatorname{supp}(d \lambda),
$$

such that

$$
\int_{\mathbb{Q}} \pi_{k, \sigma}(t) \prod_{\nu=1}^{n}\left(t-\tau_{\nu}^{(n, \sigma)}\right)^{2 s_{\nu}+1} d \lambda(t)=0, \quad k=0, \ldots, n-1 .
$$

These polynomials are called $\sigma$-orthogonal polynomials and they correspond to the sequence $\sigma=\left(s_{1}, s_{2}, \ldots\right)$. We shall often write simply $\tau_{\nu}$ or $\tau_{v}^{(n)}$ instead of $\tau_{v}^{(n, \sigma)}$. If we have $\sigma=(s, s, \ldots)$, the above polynomials reduce to the $s$-orthogonal polynomials.

An iterative process for computing the coefficients of $s$-orthogonal polynomials in a special case, when the interval $[a, b]$ is symmetric with respect to the origin and the weight $\omega$ (in the case $d \lambda(t)=\omega(t) d t$ on $[a, b]$ ) is an even function, was proposed by Vincenti [21]. He applied his process to the Legendre case. When $n$ and $s$ increase, the process becomes numerically unstable.

In [12] (see also [8]) a numerical procedure for stably calculating the nodes $\tau_{v}$ in (1.1) was proposed. In [8] a numerical procedure for stably calculating the coefficients $A_{i . v}$ in (1.1) was also proposed. Some alternative methods were proposed in $[11,19]$ and [14] (see also [18]). In [15] the methods from [8, 14] for calculating the coefficients $A_{i, v}$ in (1.1) were generalized to be able to handle those in (1.4). A simple numerical method for stably calculating the nodes $\tau_{v}$ in (1.4) has been considered recently in [13]. For all calculations in this paper we shall use the methods from $[13,15]$.

\section{Quadrature formulae of Radau and Lobatto type connected to $\sigma$-orthogonal polynomials}

Let $[a, b]$ be the support of the nonnegative measure $d \psi(t)=w(t) d t$, where $w(t)$ is the weight function.

Let

$$
\int_{a}^{b} u(t) d \psi(t)=\sum_{k=0}^{p} \alpha_{k} u^{(k)}(a)+\sum_{v=1}^{n} \sum_{i=0}^{2 s_{v}} A_{i, v}^{R} u^{(i)}\left(\tau_{v}\right)+R_{n, p}^{R},
$$

$\tau_{v} \in(a, b),-\infty<a<\infty, p \in N_{0}$, with

$$
R_{n, p}^{R}(u ; d \psi)=0 \quad \text { for } u \in \mathscr{P}_{2\left(\sum_{v=1}^{n} s_{v}+n\right)+p},
$$

be the generalized Chakalov-Popoviciu quadrature formula of Radau type. 
Let

$$
\int_{a}^{b} u(t) d \psi(t)=\sum_{k=0}^{p} \alpha_{k} u^{(k)}(a)+\sum_{k=0}^{q} \beta_{k} u^{(k)}(b)+\sum_{\nu=1}^{n} \sum_{i=0}^{2 s_{\nu}} A_{i, \nu}^{L} u^{(i)}\left(\tau_{\nu}\right)+R_{n, p, q}^{L},
$$

$\tau_{v} \in(a, b),-\infty<a<b<\infty, p, q \in N_{0}$, with

$$
R_{n, p, q}^{L}(u ; d \psi)=0 \text { for } u \in \mathscr{P}_{2\left(\sum_{v=1}^{n} s_{v}+n\right)+p+q+1},
$$

be the generalized Chakalov-Popoviciu quadrature formula of Lobatto type.

With $\mathscr{P}_{k}$ we denote the set of all polynomials of degree at most $k, k \in N_{0}$.

By using the results of Ghizzetti and Ossicini [9], we shall prove the existence and the uniqueness of the formula (2.2).

We shall denote by $\mathscr{L}[a, b]$ the class of Lebesgue-integrable (summable) functions in $[a, b]$ and by $A C^{k}[a, b]$ the class of functions whose $k$-th derivative is absolutely continuous in $[a, b], k=0,1,2, \ldots$.

Let us consider in $[a, b]$ a linear differential operator of order $L, L=1,2,3, \ldots$,

$$
E=E_{L}=\sum_{k=0}^{L} a_{k}(t) \frac{d^{L-k}}{d t^{L-k}}
$$

with the following conditions on the coefficients $a_{k}(t)$ :

$$
a_{0}(t)=1 ; \quad a_{k}(t) \in A C^{L-k-1}[a, b], k=1,2, \ldots, L-1 ; \quad a_{L}(t) \in \mathscr{L}[a, b] .
$$

The operator $E$ can be applied to the functions $u(t) \in A C^{L-1}[a, b]$, obtaining the function (defined almost everywhere):

$$
E[u(t)]=E(u)=\sum_{k=0}^{L} a_{k}(t) u^{(L-k)}(t) \in \mathscr{L}[a, b] .
$$

We associate with the operator $E$ the reduced operators

$$
E_{r}=\sum_{k=0}^{r} a_{k}(t) \frac{d^{r-k}}{d t^{r-k}}, \quad r=0,1, \ldots, L-1,
$$

and their so-called adjoint operators

where $E_{L}^{*}=E^{*}$.

$$
E_{r}^{*}=\sum_{k=0}^{r}(-1)^{r-k} \frac{d^{r-k}}{d t^{r-k}} a_{k}(t), \quad r=0,1, \ldots, L
$$

Let $K(t, \xi)$ be the so-called Cauchy resolvent kernel, which is (as a function of $t$ ) the particular solution of the homogeneous equation $E(u)=0$ which satisfies, at the point $\xi$, the initial conditions:

$$
\left[\frac{\partial^{h}}{\partial t^{h} K(t, \xi)}\right]_{t=\xi}=\delta_{h, L-1}, \quad h=0,1, \ldots, L-1,
$$


where

$$
\delta_{r s}= \begin{cases}0, & r \neq s \\ 1, & r=s\end{cases}
$$

Let us consider the elementary quadrature formula

$$
\int_{a}^{b} u(t) d \psi(t)=\sum_{h=0}^{L-1} \sum_{i=1}^{l} C_{h i} u^{(h)}\left(x_{i}\right)+R(u), \quad[E(u)=0 \Rightarrow R(u)=0]
$$

where $E$ is the linear differential operator of order $L$.

In [9, pp. 29-31] the following result is proved.

THEOREM 2.1. If, having $l$ fixed nodes $x_{1}, x_{2}, \ldots, x_{l}$ and $l L$ constants $C_{h i}$, the linear functional

$$
R(u)=\int_{a}^{b} u(t) w(t) d t-\sum_{h=0}^{L-1} \sum_{i=1}^{l} C_{h i} u^{(h)}\left(x_{i}\right)
$$

is null when $u$ is a solution of the homogeneous linear differential equation $E(u)=0$, then there are $l-1$ uniquely determined solutions $\varphi_{1}(t), \ldots, \varphi_{l-1}(t)$ of the differential equation $E^{*}(\varphi)=w$ which, together with $\varphi_{0}(t)$ and $\varphi_{l}(t)$ given by

$$
\varphi_{0}(t)=-\int_{a}^{t} K(\xi, t) w(\xi) d \xi, \quad \varphi_{l}(t)=\int_{t}^{b} K(\xi, t) w(\xi) d \xi
$$

validate

$$
C_{h i}=\left\{E_{L-h-1}^{*}\left[\varphi_{i}(t)-\varphi_{i-1}(t)\right]\right\}_{t=x_{i}} ; \quad h=0,1, \ldots, L-1, i=1,2, \ldots, l,
$$

and

$$
R[u(t)]=\sum_{i=0}^{l} \int_{x_{i}}^{x_{i+1}} \varphi_{i}(t) E[u(t)] d t
$$

Having fixed the nodes $x_{1}, x_{2}, \ldots, x_{l}$ and the linear differential operator $E$, we may write the quadrature formula $(2.3)$ in $\infty^{(l-1) L}$ different ways, since $(l-1) L$ is the number of arbitrary constants on which the $l-1$ solutions $\varphi_{1}(t), \ldots, \varphi_{l-1}(t)$ of the differential equation $E^{*}(\varphi)=w$ of order $L$ depend.

Define the generalized Gauss problem (see [9, pp. 41-45]).

The question is whether, having fixed nonnegative integers $p_{i}\left(p_{i} \leq L-1\right)$, $i=1, \ldots, l$, with $(\exists i=1, \ldots, l) p_{i} \geq 1$, it is possible to make use of the arbitrary nature of these parameters to drop the values $u^{(h)}\left(x_{i}\right)$ of the derivatives of order higher 
than $L-p_{i}-1, i=1, \ldots, l$, from (2.3), that is, whether there can exist a formula of the type

$$
\int_{a}^{b} u(t) d \psi(t)=\sum_{i=1}^{l} \sum_{h=0}^{L-p_{i}-1} C_{h i} u^{(h)}\left(x_{i}\right)+R(u), \quad[E(u)=0 \Rightarrow R(u)=0] .
$$

The answer is given by the following theorem (see [9, Problem 2, p. 45]), which can be proved similarly to Theorem 2.5 .I in [9].

THEOREM 2.2. Given the nodes $x_{1}, \ldots, x_{l}$, which satisfy

$$
a \leq x_{1}<x_{2}<\cdots<x_{1} \leq b,
$$

the linear differential operator $E$ of order $L$ and nonnegative integers $p_{i}\left(p_{i} \leq L-1\right)$, $i=1, \ldots, l$, with $(\exists i=1, \ldots, l) p_{i} \geq 1$, consider the homogeneous differential problem

$$
E(u)=0 ; \quad u^{(h)}\left(x_{i}\right)=0, \quad h=0,1, \ldots, L-p_{i}-1, i=1, \ldots, l .
$$

If this problem has no non-trivial solutions [whence $L \leq l L-\sum_{i=1}^{l} p_{i}$ ] it is possible to write a quadrature formula of the type (2.4) in $\infty^{l L-\sum_{i=1}^{\prime} p_{i}-L}$ different ways. If on the other hand the problem (2.6) has $q$ linearly independent solutions $U_{j}(t)[j=$ $1,2, \ldots, q$, with $\left.L-L l+\sum_{i=1}^{l} p_{i} \leq q \leq p_{i}(\forall i=1, \ldots, l) ; 1 \leq q\right]$ then (2.4) may apply only if the $q$ conditions

$$
\int_{a}^{b} U_{j}(t) d \psi(t)=0, \quad j=1, \ldots, q,
$$

are satisfied; if so, there are $\infty^{L-\sum_{i=1}^{\prime} p_{i}-L+q}$ possible formulae of form (2.4).

Consider (2.2), with conditions (2.5) for

$$
\begin{gathered}
x_{1}=a, \quad x_{v+1}=\tau_{v}, \nu=1, \ldots, n, \quad x_{l}=x_{n+2}=b, \\
\left(\text { where } \quad C_{h 1}=\alpha_{h}, C_{h i}=A_{h, i}^{L}, C_{h l}=C_{h, n+2}=\beta_{h}\right)
\end{gathered}
$$

for which $R(u)=0, \forall u \in \mathscr{P}_{2\left(\sum_{v=1}^{n} s_{v}+n\right)+p+q+1}$.

Let $L=2\left(\sum_{v=1}^{n} s_{v}+n\right)+p+q+2$. By virtue of Theorem 2.2 we must consider the boundary problem

with

$$
d^{L} u / d t^{L}=0
$$

$$
\begin{gathered}
u^{(h)}(a)=0, \quad h=0, \ldots, p ; \quad u^{(h)}(b)=0, \quad h=0, \ldots, q \\
u^{(h)}\left(\tau_{v}\right)=0, \quad h=0, \ldots, 2 s_{v}, v=1, \ldots, n
\end{gathered}
$$


and its non trivial solutions which are

$$
t^{k}(t-a)^{p+1}(b-t)^{q+1} \prod_{\nu=1}^{n}\left(t-\tau_{v}\right)^{2 s_{\nu}+1}, \quad k=0,1, \ldots, n-1 .
$$

Therefore, (2.2) is possible if and only if

$$
\int_{a}^{b}(t-a)^{p+1}(b-t)^{q+1} \cdot t^{k} \prod_{\nu=1}^{n}\left(t-\tau_{\nu}\right)^{2 s_{\nu}+1} d \psi(t)=0, \quad k=0,1, \ldots, n-1,
$$

are satisfied and this shows that the nodes $\tau_{\nu}$ must coincide with the zeros of the polynomial $\pi_{n, \sigma}(t)$ of the $\sigma$-orthogonal system relative to the measure

$$
(t-a)^{p+1}(b-t)^{q+1} d \psi(t) .
$$

With such a choice of the nodes (2.2) is unique since, with the notation of Theorem 2.2 , we have

$$
\begin{aligned}
l L-\sum_{i=1}^{l} p_{i}-L+q= & +q+2+\sum_{v=1}^{n}\left(2 s_{v}+1\right) \\
& -\left[2\left(\sum_{\nu=1}^{n} s_{v}+n\right)+p+q+2\right]+n=0 .
\end{aligned}
$$

Similarly, we can conclude that (2.1) exists and it is necessarily unique. In the following, we shall put $p=m=q$, without loss of generality.

\section{Calculation of the formulae (2.1), (2.2)}

We give two lemmas, which give a connection between the generalized ChakalovPopoviciu quadrature (1.4) and the corresponding formulae of Radau and Lobatto type.

LEMMA 3.1. If the measure $d \psi(t)$ admits ${ }^{*}$ the generalized Chakalov-Popoviciu quadrature of Lobatto type (2.2) (in which $p=q=m$ ), with distinct real zeros $\tau_{v}=\tau_{v}^{(n)}=\tau_{v}^{(n, \sigma)}, v=1, \ldots, n$, all contained in the open interval $(a, b)$, there exists then a generalized Chakalov-Popoviciu formula

$$
\int_{a}^{b} g(t) d \lambda(t)=\sum_{\nu=1}^{n} \sum_{i=0}^{2 s_{v}} A_{i, \nu}^{G} g^{(i)}\left(\tau_{\nu}^{(n)}\right)+R_{n}^{G}(g),
$$

*For example, this holds if $d \psi(t)$ is nonnegative (or nonpositive). 
where $d \lambda(t)=[(b-t)(t-a)]^{m+1} d \psi(t)$, the nodes $\tau_{\nu}^{(n)}$ are the zeros of $\sigma$-orthogonal polynomial $\pi_{n, \sigma}(\cdot ; d \lambda)$, while the weights $A_{i, v}^{G}$ are expressible in terms of those in (2.2) by

$$
A_{i, v}^{G}=\sum_{k=i}^{2 s_{v}}\left(\begin{array}{l}
k \\
i
\end{array}\right)\left[((b-t)(t-a))^{m+1}\right]_{t=\tau_{v}}^{(k-i)} A_{k, v}^{L},
$$

where $i=0, \ldots, 2 s_{v}, v=1, \ldots, n$.

PROOF. Let $g(t)=((b-t)(t-a))^{m+1} p(t), p \in \mathscr{P}_{2\left(\sum_{v=1}^{n} s_{v}+n\right)-1}$ and $\tau_{v}=\tau_{v}^{(n)}$. We have by (2.2)

$$
\int_{a}^{b} g(t) d \psi(t)=\sum_{\nu=1}^{n} \sum_{k=0}^{2 s_{\nu}}\left[((b-t)(t-a))^{m+1} p(t)\right]_{t=\tau_{\nu}}^{(k)} A_{k, \nu}^{L},
$$

and by (3.1)

$$
\int_{a}^{b} p(t) d \lambda(t)=\sum_{\nu=1}^{n} \sum_{i=0}^{2 s_{\nu}} A_{i, \nu}^{G} p^{(i)}\left(\tau_{\nu}\right)
$$

So, we have that

$$
\sum_{\nu=1}^{n} \sum_{k=0}^{2 s_{v}}\left[((b-t)(t-a))^{m+1} p(t)\right]_{t=\tau_{\nu}}^{(k)} A_{k, \nu}^{L}=\sum_{\nu=1}^{n} \sum_{i=0}^{2 s_{\nu}} A_{i, \nu}^{G} p^{(i)}\left(\tau_{v}\right) .
$$

Applying the Leibniz formula to the $k$-th derivative in the second sum, we find

$$
\begin{aligned}
\sum_{k=0}^{2 s_{v}} & {\left[((b-t)(t-a))^{m+1} p(t)\right]_{t=\tau_{v}}^{(k)} A_{k, \nu}^{L} } \\
& =\sum_{k=0}^{2 s_{v}}\left[\sum_{i=0}^{k}\left(\begin{array}{l}
k \\
i
\end{array}\right)\left(((b-t)(t-a))^{m+1}\right)^{(k-i)} p^{(i)}(t)\right]_{t=\tau_{v}} A_{k, \nu}^{L} \\
& =\sum_{i=0}^{2 s_{v}}\left(\sum_{k=i}^{2 s_{v}}\left(\begin{array}{l}
k \\
i
\end{array}\right)\left(((b-t)(t-a))^{m+1}\right)_{t=\tau_{v}}^{(k-i)} A_{k, \nu}^{L} p^{(i)}\left(\tau_{\nu}\right)\right)=\sum_{i=0}^{2 s_{v}} A_{i, \nu}^{G} p^{(i)}\left(\tau_{\nu}\right),
\end{aligned}
$$

where

$$
A_{i, v}^{G}=\sum_{k=i}^{2 s_{v}}\left(\begin{array}{c}
k \\
i
\end{array}\right)\left[((b-t)(t-a))^{m+1}\right]_{t=\tau_{v}}^{(k-i)} A_{k, v}^{L} ; i=0, \ldots, 2 s_{v}, v=1, \ldots, n .
$$

Similarly we can prove the following lemma.

LEMMA 3.2. If the measure $d \psi(t)$ admits the generalized Chakalov-Popoviciu quadrature of Radau type (2.1) (in which $p=m$ ), with distinct real zeros $\tau_{\nu}=\tau_{\nu}^{(n) *}$, 
TABLE 4.1.

\begin{tabular}{|c|c|c|}
\hline$v$ & $\tau_{2 v-1}$ & $\tau_{2 v}$ \\
\hline 1 & $8.06063896919729(-02)$ & $2.42198578093389(-01)$ \\
\hline 2 & $4.93117605175704(-01)$ & $7.15377067743040(-01)$ \\
\hline 3 & $8.94837669670698(-01)$ & \\
\hline
\end{tabular}

$v=1, \ldots, n$, all contained in the open interval $(a, b)$, there exists then a generalized Chakalov-Popoviciu formula (3.1), where $d \lambda(t)=d \lambda^{*}(t)=(t-a)^{m+1} d \psi(t)$, the nodes $\tau_{\nu}^{(n) *}$ are the zeros of $\sigma$-orthogonal polynomial $\pi_{n, \sigma}\left(\cdot ; d \lambda^{*}\right)$, while the weights $A_{i, \nu}^{G}$ are expressible in terms of those in (3.1) by

$$
A_{i, \nu}^{G}=\sum_{k=i}^{2 s_{v}}\left(\begin{array}{c}
k \\
i
\end{array}\right)\left[(t-a)^{m+1}\right]_{t=\tau_{v}}^{(k-i)} A_{k, \nu}^{R} ; \quad i=0, \ldots, 2 s_{v}, v=1, \ldots, n .
$$

We can write the triangular system (3.2) in the form

$$
A_{i, \nu}^{G}=\sum_{k=i}^{2 s_{v}} C_{k}^{(i, \nu)} A_{k, \nu}^{L} ; \quad i=0, \ldots, 2 s_{\nu}, \nu=1, \ldots, n,
$$

where

$$
\begin{aligned}
C_{k}^{(i, v)} & =\left(\begin{array}{c}
k \\
i
\end{array}\right)\left[((b-t)(t-a))^{m+1}\right]_{t=\tau_{v}}^{(k-i)} \\
& = \begin{cases}0 ; & k<i, \\
\frac{k !}{i !} \sum_{l=0}^{k-i} \frac{(-1)^{l}(m+1) !^{2}\left(\tau_{\nu}-a\right)^{m-k+i+l+1}\left(b-\tau_{\nu}\right)^{m-l+1}}{l !(k-i-l) !(m-k+i+l+1) !(m-l+1) !} ; & i \leq k \leq 2 s_{\nu} .\end{cases}
\end{aligned}
$$

The triangular system (3.3) we can write in the form

$$
A_{i, \nu}^{G}=\sum_{k=i}^{2 s_{v}} B_{k}^{(i, \nu)} A_{k, v}^{R} ; \quad i=0, \ldots, 2 s_{v}, \nu=1, \ldots, n,
$$

where

$$
B_{k}^{(i, v)}=\left(\begin{array}{l}
k \\
i
\end{array}\right)\left[(t-a)^{m+1}\right]_{t=\tau_{v}}^{(k-i)}= \begin{cases}0 ; & k<i, \\
\frac{k !(m+1) !\left(\tau_{\nu}-a\right)^{m-k+i+1}}{i !(k-i) !(m-k+i+1) !} ; & i \leq k \leq 2 s_{\nu} .\end{cases}
$$

\section{Numerical results}

As an example we consider the Chebyshev measure $d \psi(t)=d t / \sqrt{t-t^{2}}$ on the interval $I=[a, b]=[0,1]$ in the Lobatto case. Therefore we have

$$
d \lambda(t)=[t(1-t)]^{m+1 / 2} d t .
$$


In Table 4.1 the nodes $\tau_{v}$ of the corresponding Chakalov-Popoviciu quadrature formula (1.4), for $\sigma=(0,3,1,2,1), n=5$, are given.

TABLE 4.2.

\begin{tabular}{|c|c|c|c|}
\hline$\nu$ & $i$ & $A_{i, v}^{G}$ & $A_{i+1, v}^{G}$ \\
\hline 1 & 0 & $4.20127478080609(-08)$ & \\
\hline 2 & 0 & $3.71485589869411(-05)$ & $2.53189264911106(-06)$ \\
2 & 2 & $1.24288590234291(-07)$ & $3.28295940614803(-09)$ \\
2 & 4 & $6.72398482227105(-11)$ & $7.51024105924184(-13)$ \\
2 & 6 & $6.18123581366015(-15)$ & \\
\hline 3 & 0 & $9.25967832748324(-05)$ & $1.88049797773032(-08)$ \\
3 & 2 & $9.57294036599511(-08)$ & \\
\hline 4 & 0 & $4.27128390332233(-05)$ & $-1.71275165622089(-06)$ \\
4 & 2 & $7.93022775662744(-08)$ & $-1.08954169181538(-09)$ \\
4 & 4 & $1.92447787210554(-11)$ & \\
\hline 5 & 0 & $5.22053028280481(-07)$ & $-1.15793712000017(-08)$ \\
5 & 2 & $1.12436028390154(-10)$ & \\
\hline
\end{tabular}

In Table 4.2 the weights $A_{i, v}^{G}$ of the corresponding Chakalov-Popoviciu quadrature formula are given. For $m=5$, the weights $A_{i, \nu}^{L}$ of the corresponding ChakalovPopoviciu quadrature formula of Lobatto type (2.2) are given in Table 4.3.

TABLE 4.3.

\begin{tabular}{|c|c|c|c|}
\hline$\nu$ & $i$ & $A_{i, \nu}^{L}$ & $A_{i+1, \nu}^{L}$ \\
\hline 1 & 0 & $2.53603580873942(-01)$ & \\
\hline 2 & 0 & $6.54607056346764(-01)$ & $2.47009978449190(-03)$ \\
2 & 2 & $1.78916012822395(-03)$ & $8.68913193385365(-06)$ \\
2 & 4 & $1.06575641867557(-06)$ & $3.29355080757672(-09)$ \\
2 & 6 & $1.61701214701959(-10)$ & \\
\hline 3 & 0 & $3.98578546685041(-01)$ & $-1.82300441012789(-04)$ \\
3 & 2 & $3.92553687612449(-04)$ & \\
\hline 4 & 0 & $5.24003817562713(-01)$ & $-8.43880698485214(-04)$ \\
4 & 2 & $9.30751562588805(-04)$ & $-1.57766077104084(-06)$ \\
4 & 4 & $2.70074453090533(-07)$ & \\
\hline 5 & 0 & $4.11726824044766(-01)$ & $-3.70334318380999(-04)$ \\
5 & 2 & $1.61911889209916(-04)$ & \\
\hline
\end{tabular}

Table 4.4 gives the corresponding coefficients $\alpha_{k}, \beta_{k}$ in the endpoints $-1,1$. The numbers in parentheses denote decimal exponents. The programs were realized in double precision arithmetic in FORTRAN. 
TABLE 4.4 .

\begin{tabular}{|c|c|r|}
\hline$k$ & $\alpha_{k}$ & \multicolumn{1}{|c|}{$\beta_{k}$} \\
\hline 0 & $4.48079461557622(-01)$ & $4.50993366518945(-01)$ \\
1 & $6.76966763724565(-03)$ & $-6.86234369124486(-03)$ \\
2 & $7.83092608702163(-05)$ & $7.94301775592061(-05)$ \\
3 & $5.74636703570962(-07)$ & $-5.80256392257038(-07)$ \\
4 & $2.44687263671571(-09)$ & $2.45051821492370(-09)$ \\
5 & $4.67095320822040(-12)$ & $-4.62776252162197(-12)$ \\
\hline
\end{tabular}

TABLE 4.5 .

\begin{tabular}{|c|c|c|c|}
\hline$n$ & $\sigma$ & $m$ & $R e$ \\
\hline 2 & $(1,1)$ & 0 & $1.0(-09)$ \\
\hline 2 & $(0,2)$ & 1 & $3.6(-12)$ \\
\hline 2 & $(0,3)$ & 1 & $9.9(-15)$ \\
\hline 3 & $(1,0,1)$ & 0 & $1.6(-12)$ \\
\hline 3 & $(0,1,2)$ & 0 & $4.8(-15)$ \\
\hline 3 & $(0,1,2)$ & 1 & $6.6(-16)$ \\
\hline
\end{tabular}

By using (2.2) and the presented methods we have calculated the integral

$$
J=\int_{0}^{1} \frac{e^{2 t}}{\sqrt{t-t^{2}}} d t=10.8118661043980 \ldots,
$$

for some $n, \sigma, m$. In Table 4.5 the relative errors $R e$ of these calculations are given.

\section{An application-Moment-preserving spline approximation with variable defects on finite intervals}

Let $z_{+}^{i}$ be $z^{i}$, if $z \geq 0$, and 0 , if $z<0$.

In this section we discuss the case of approximating a function $f=f(t)$ on some given finite interval $I=[a, b]$, which can be standardized to $[a, b]=[0,1]$, by a spline function of degree $m \geq 2$ and defects $d_{v}\left(1 \leq d_{v} \leq m, v=1, \ldots, n\right)$, with $n$ knots. Under suitable assumptions on $f$ and $d_{v}=2 s_{\nu}+1, v=1, \ldots, n$, we shall show that our problem has a unique solution if and only if certain generalized ChakalovPopoviciu quadrature formulae of Radau and Lobatto type exist corresponding to measures depending on $f$. Existence, uniqueness and pointwise convergence are assured if $f$ is completely monotonic on $[0,1]$.

Spline approximation on [0,1]. A spline function of degree $m \geq 2$ and defects $d_{v}$, $v=1, \ldots, n$, with $n$ (distinct) knots $\tau_{1}, \ldots, \tau_{n}$ in the interior of $[0,1]$, can be written 
in terms of truncated powers in the form

$$
s_{n, m}(t)=p_{m}(t)+\sum_{v=1}^{n} \sum_{i=m-d_{v}+1}^{m} a_{i, v}\left(\tau_{v}-t\right)_{+}^{i},
$$

where $a_{i, v}$ are real numbers and $p_{m}(t)$ is a polynomial of degree $\leq m$.

Similarly as in [5] we shall consider two related problems.

PROBLEM I. Determine $s_{n, m}$ in (5.1) such that

$$
\int_{0}^{1} t^{j} s_{n, m}(t) d t=\int_{0}^{1} t^{j} f(t) d t, \quad j=0,1, \ldots, \sum_{\nu=1}^{n} d_{\nu}+n+m .
$$

PROBLEM I*. Determine $s_{n, m}$ in (5.1) such that

$$
s_{n, m}^{(k)}(1)=p_{m}^{(k)}(1)=f^{(k)}(1), \quad k=0, \ldots, m,
$$

and such that (5.2) holds for $j=0,1, \ldots, \sum_{v=1}^{n} d_{\nu}+n-1$.

In this section we shall reduce our problems to $\sigma$-orthogonality and generalized Chakalov-Popoviciu quadratures by restricting the class of functions $f$.

In order to reduce our problems (5.2) and (5.3) to $\sigma$-orthogonality, we have to put $d_{v}=2 s_{v}+1, v=1, \ldots, n$, that is, the defects of the spline function (5.1) should be odd.

Let

$$
\varphi_{k}=\frac{(-1)^{k}}{m !} f^{(k)}(1), \quad b_{k}=\frac{(-1)^{k}}{m !} p_{m}^{(k)}(1), \quad k=0, \ldots, m .
$$

Applying $m+1$ integration by parts to the integrals in the moment equation (5.2) we obtain (see [5])

$$
\begin{array}{r}
\sum_{k=0}^{m} b_{k}\left[t^{m+1+j}\right]_{t=1}^{(m-k)}+\sum_{v=1}^{n} \sum_{i=m-2 s_{v}}^{m} a_{i, v} t_{\nu}^{j+i+1} \frac{i !(m+j+1) !}{m !(j+i+1) !} \\
=\sum_{k=0}^{m} \varphi_{k}\left[t^{m+1+j}\right]_{t=1}^{(m-k)}+\frac{(-1)^{m+1}}{m !} \int_{0}^{1} t^{m+1+j} f^{(m+1)}(t) d t,
\end{array}
$$

where $j=0,1, \ldots, 2\left(\sum_{v=1}^{n} s_{v}+n\right)+m$.

For the second sum in (5.5) we may observe that

$$
\sum_{\nu=1}^{n} \sum_{i=m-2 s_{\nu}}^{m} a_{i, \nu} \tau_{\nu}^{j+i+1} \frac{i !(m+j+1) !}{m !(j+i+1) !}=\sum_{\nu=1}^{n} \sum_{i=m-2 s_{\nu}}^{m} \frac{i !}{m !} a_{i, \nu}\left[t^{m+j+1}\right]_{t=\tau_{\nu}}^{(m-i)} .
$$


Changing indices $(k=m-i)$, the second sum on the right becomes

$$
\sum_{k=0}^{2 s_{v}} \frac{(m-k) !}{m !} a_{m-k, \nu}\left[t^{m+1} t^{j}\right]_{t=\tau_{v}}^{(k)},
$$

hence defining the measure

$$
d \psi(t)=\frac{(-1)^{m+1}}{m !} f^{(m+1)}(t) d t \quad \text { on } \quad[0,1]
$$

Equation (5.5) may be rewritten

$$
\begin{gathered}
\sum_{k=0}^{m} b_{k}\left[t^{m+1+j}\right]_{t=1}^{(m-k)}+\sum_{\nu=1}^{n} \sum_{k=0}^{2 s_{v}} \frac{(m-k) !}{m !} a_{m-k, v}\left[t^{m+1+j}\right]_{t=\tau_{v}}^{(k)} \\
=\sum_{k=0}^{m} \varphi_{k}\left[t^{m+1+j}\right]_{t=1}^{(m-k)}+\int_{0}^{1} t^{m+1+j} d \psi(t)
\end{gathered}
$$

where $j=0,1, \ldots, 2\left(\sum_{\nu=1}^{n} s_{v}+n\right)+m$.

Now we can state the main result for Problem I.

THEOREM 5.1. Let $f \in C^{m+1}[0,1]$. There exists a unique spline function (5.1) on $[0,1]$, with $d_{v}=2 s_{v}+1, v=1, \ldots, n$, satisfying (5.2) if and only if the measure $d \psi(t)$ in (5.7) admits a generalized Chakalov-Popoviciu quadrature of Lobatto type

$$
\begin{aligned}
\int_{0}^{1} g(t) d \psi(t)= & \sum_{k=0}^{m}\left[\alpha_{k} g^{(k)}(0)+\beta_{k} g^{(k)}(1)\right] \\
& +\sum_{\nu=1}^{n} \sum_{i=0}^{2 s_{\nu}} A_{i, \nu}^{L} g^{(i)}\left(\tau_{\nu}^{(n)}\right)+R_{n, m}^{L}(g ; d \psi),
\end{aligned}
$$

where

$$
R_{n, m}^{L}(g ; d \psi)=0 \text { for } g \in \mathscr{P}_{2\left(\sum_{v=1}^{n} s_{v}+n+m\right)+1},
$$

with distinct real zeros $\tau_{v}^{(n)}, v=1, \ldots, n$, all contained in the open interval $(0,1)$. The spline function in (5.1) is given by

$$
\tau_{v}=\tau_{v}^{(n)}, \quad a_{m-k, v}=\frac{m !}{(m-k) !} A_{k, v}^{L} ; \quad v=1, \ldots, n, k=0, \ldots, 2 s_{v},
$$

where $\tau_{v}^{(n)}$ are the interior nodes of the generalized Chakalov-Popoviciu quadrature formula of Lobatto type and $A_{k, \nu}^{L}$ are the corresponding weights, while the polynomial $p_{m}(t)$ is given by

$$
p_{m}^{(k)}(1)=f^{(k)}(1)+(-1)^{k} m ! \beta_{m-k}, \quad k=0,1, \ldots, m,
$$

where $\beta_{m-k}$ is the coefficient of $g^{(m-k)}(1)$ in (5.9). 
PROOF. Putting $g(t)=t^{m+1} p(t), p \in \mathscr{P}_{2\left(\sum_{v=1}^{n} s_{v}+n\right)+m}$, in (5.9) and noting (5.10) yields, for every $p \in \mathscr{P}_{2\left(\sum_{v=1}^{n} s_{v}+n\right)+m}$,

$$
\sum_{k=0}^{m} \beta_{k}\left[t^{m+1} p(t)\right]_{t=1}^{(k)}+\sum_{\nu=1}^{n} \sum_{i=0}^{2 s_{v}} A_{i, \nu}^{L}\left[t^{m+1} p(t)\right]_{t=\tau_{v}}^{(k)}=\int_{0}^{1} t^{m+1} p(t) d \psi(t),
$$

which is identical to (5.8), if we identify

$$
\begin{array}{ll}
b_{m-k}-\varphi_{m-k}=\beta_{k}, & k=0,1, \ldots, m ; \\
a_{m-k, v}=\frac{m !}{(m-k) !} A_{k, \nu}^{L}, & \nu=1, \ldots, n, k=0, \ldots, 2 s_{\nu} .
\end{array}
$$

REMARK A. The case $s_{1}=\cdots=s_{n}=0$ of Theorem 5.1 has been obtained in [5], and generalized in [6] to the case $s_{1}=\cdots=s_{n}=s, s \in N$.

If $f$ is completely monotonic on $[0,1]$ then $d \psi(t)$ in (5.7) is a positive measure for every $m$, and then by virtue of the assumptions in Theorem 5.1 the generalized Chakalov-Popoviciu quadrature formula of Lobatto type exists uniquely, with $n$ distinct real nodes $\tau_{\nu}^{(n)}$ in $(0,1)$.

The solution of Problem $I^{*}$ can be given in a similar way.

THEOREM 5.2. Let $f \in C^{m+1}[0,1]$. There exists a unique spline function on $[0,1]$,

$$
s_{n, m}^{*}(t)=p_{m}^{*}(t)+\sum_{\nu=1}^{n} \sum_{i=m-2 s_{v}}^{m} a_{i, \nu}^{*}\left(\tau_{\nu}^{*}-t\right)_{+}^{i}, \quad \begin{aligned}
& 0<\tau_{\nu}^{*}<1, \\
& \tau_{\nu}^{*} \neq \tau_{\mu}^{*} \text { for } v \neq \mu,
\end{aligned}
$$

satisfying (5.3) and (5.2), for $j=0,1, \ldots, 2\left(\sum_{v=1}^{n} s_{\nu}+n\right)-1$, if and only if the measure $d \psi(t)$ in (5.7) admits a generalized Chakalov-Popoviciu quadrature of Radau type

$$
\int_{0}^{1} g(t) d \psi(t)=\sum_{k=0}^{m} \alpha_{k}^{*} g^{(k)}(0)+\sum_{\nu=1}^{n} \sum_{i=0}^{2 s_{\nu}} A_{i, \nu}^{R} g^{(i)}\left(\tau_{\nu}^{(n) *}\right)+R_{n, m}^{R}(g ; d \psi),
$$

where

$$
R_{n, m}^{R}(g ; d \psi)=0 \text { for } g \in \mathscr{P}_{2\left(\sum_{v=1}^{n} s_{v}+n\right)+m},
$$

with distinct real zeros $\tau_{v}^{(n) *}, v=1, \ldots, n$, all contained in the open interval $(0,1)$. The knots $\tau_{v}^{*}$ in (5.13) are then precisely these zeros,

$$
\tau_{\nu}^{*}=\tau_{\nu}^{(n) *}, \quad v=1, \ldots, n
$$


and

$$
a_{m-k, \nu}^{*}=\frac{m !}{(m-k) !} A_{k, \nu}^{R} ; \quad v=1, \ldots, n, k=0, \ldots, 2 s_{v},
$$

while the polynomial $p_{m}^{*}(t)$ is given by

$$
p_{m}^{*}(t)=\sum_{k=0}^{m} \frac{f^{(k)}(1)}{k !}(t-1)^{k} .
$$

REMARK B. Therefore, by using our methods from $[13,15]$, the results from Section 3 , and the formulae (5.11) and (5.12), or (5.15)-(5.17), we can easily determine the spline approximation $s_{n, m}(t)$, or $s_{n, m}^{*}(t)$, respectively.

Error analysis. Similarly as in [5], following [7], we can prove the following statement regarding the error of spline approximations.

THEOREM 5.3. Define $r_{x}(t)=(t-x)_{+}^{m}, 0 \leq t \leq 1$. Under the conditions of Theorems 5.1 and 5.2, we have

$$
f(x)-s_{n, m}(x)=R_{n, m}^{L}\left(r_{x} ; d \psi\right), \quad 0<x<1,
$$

and

$$
f(x)-s_{n, m}^{*}(x)=R_{n, m}^{R}\left(r_{x} ; d \psi\right), \quad 0<x<1,
$$

respectively, where $R_{n, m}^{L}(g ; d \psi)$ and $R_{n, m}^{R}(g ; d \psi)$ are the remainder terms in the corresponding Chakalov-Popoviciu formulae of Lobatto and Radau type.

PROOF. We shall prove (5.18). As in [5] we have

$$
f(x)=\sum_{k=0}^{m} \frac{f^{(k)}(1)}{k !}(x-1)^{k}+\int_{0}^{1} r_{x}(t) d \psi(t) .
$$

By (5.11)

$$
s_{n, m}(x)=\sum_{k=0}^{m} \frac{p_{m}^{(k)}(1)}{k !}(x-1)^{k}+\sum_{\nu=1}^{n} \sum_{i=m-2 s_{v}}^{m} \frac{m !}{i !} A_{m-i, \nu}^{L}\left(\tau_{\nu}-x\right)_{+}^{i}
$$

and changing indices $(k=m-i)$, the third sum on the right becomes

$$
\sum_{i=m-2 s_{v}}^{m} \frac{m !}{i !} A_{m-i, v}^{L}\left(\tau_{v}-x\right)_{+}^{i}=\sum_{k=0}^{2 s_{v}} \frac{m !}{(m-k) !} A_{k, \nu}^{L}\left(\tau_{\nu}-x\right)_{+}^{m-k}=\sum_{k=0}^{2 s_{v}} A_{k, \nu}^{L} r_{x}^{(k)}\left(\tau_{v}\right) .
$$

Equation (5.21) may be rewritten as

$$
s_{n, m}(x)=\sum_{k=0}^{m} \frac{p_{m}^{(k)}(1)}{k !}(x-1)^{k}+\sum_{\nu=1}^{n} \sum_{k=0}^{2 s_{\nu}} A_{k, \nu}^{L} r_{x}^{(k)}\left(\tau_{\nu}\right)
$$


Subtracting (5.22) from (5.20) gives

$$
\begin{aligned}
f(x)-s_{n, m}(x)= & \int_{0}^{1} r_{x}(t) d \psi(t)+\sum_{k=0}^{m} \frac{1}{k !}\left(f^{(k)}(1)-p_{m}^{(k)}(1)\right)(x-1)^{k} \\
& -\sum_{v=1}^{n} \sum_{k=0}^{2 s_{v}} A_{k, v}^{L} r_{x}^{(k)}\left(\tau_{v}\right)
\end{aligned}
$$

which, by virtue of (5.12) and (5.4), yields

$$
f(x)-s_{n, m}(x)=\int_{0}^{1} r_{x}(t) d \psi(t)-\sum_{k=0}^{m} \frac{m !}{k !} \beta_{m-k}(1-x)^{k}-\sum_{\nu=1}^{n} \sum_{k=0}^{2 s_{v}} A_{k, \nu}^{L} r_{x}^{(k)}\left(\tau_{\nu}\right) .
$$

But

$$
r_{x}^{(k)}(0)=0, \quad r_{x}^{(k)}(1)=\frac{m !}{(m-k) !}(1-x)^{m-k}, \quad k=0, \ldots, m,
$$

so that

$$
f(x)-s_{n, m}(x)=\int_{0}^{1} r_{x}(t) d \psi(t)-\sum_{k=0}^{m} \beta_{m-k} r_{x}^{(m-k)}(1)-\sum_{\nu=1}^{n} \sum_{k=0}^{2 s_{v}} A_{k, v}^{L} r_{x}^{(k)}\left(\tau_{v}\right)
$$

as claimed in (5.18).

The proof of (5.19) is entirely analogous to the proof of (5.18) and it shall be omitted.

\section{On an analytic formula for the coefficients $A_{i, v}$ in (1.4)}

Let

$$
\omega_{v}(t)=\frac{\prod_{l=1}^{n}\left(t-\tau_{l}\right)^{2 s_{l}+1}}{\left(t-\tau_{v}\right)^{2 s_{v}+1}}
$$

On the basis of Hermite's interpolation (see [1, pp. 163-173]) we obtained the weights $A_{i, v}$ in the generalized Chakalov-Popoviciu quadrature formula (1.4) (see [15])

$$
A_{i, \nu}=\frac{1}{i !} \sum_{k=0}^{2 s_{\nu}-i} \frac{1}{k !}\left[\frac{\left(t-\tau_{\nu}\right)^{2 s_{\nu}+1}}{\Omega(t)}\right]_{t=\tau_{\nu}}^{(k)} \int_{\mathbb{R}} \frac{\Omega(t)}{\left(t-\tau_{\nu}\right)^{2 s_{\nu}-i-k+1}} d \lambda(t),
$$

where

$$
\Omega(t)=\left(t-\tau_{1}\right)^{2 s_{1}+1}\left(t-\tau_{2}\right)^{2 s_{2}+1} \cdots\left(t-\tau_{n}\right)^{2 s_{n}+1}=\prod_{l=1}^{n}\left(t-\tau_{l}\right)^{2 s_{1}+1},
$$

and $i=0,1, \ldots, 2 s_{v}, v=1, \ldots, n$.

In the following statement we shall obtain an alternative expression. 
LEMMA 6.1. The coefficients $A_{i, \nu}$ in (1.4) can be expressed in the form

$$
A_{i, v}=\frac{1}{i !\left(2 s_{v}-i\right) !}\left[\frac{1}{\omega_{v}(t)} \int_{\mathbf{R}} \frac{\prod_{l=1}^{n}\left(x-\tau_{l}\right)^{2 s_{t}+1}-\prod_{l=1}^{n}\left(t-\tau_{l}\right)^{2 s_{l}+1}}{x-t} d \lambda(x)\right]_{t=\tau_{v}}^{\left(2 s_{v}-i\right)},
$$

where $i=0,1, \ldots, 2 s_{v}, v=1, \ldots, n$.

ProOF. If we put $k=2 s_{v}-i-m$ in (6.1), then we have

$$
\begin{aligned}
A_{i, \nu}= & \frac{1}{i !} \sum_{m=0}^{2 s_{\nu}-i} \frac{1}{\left(2 s_{\nu}-i-m\right) !}\left[\frac{\left(t-\tau_{\nu}\right)^{2 s_{\nu}+1}}{\prod_{l=1}^{n}\left(t-\tau_{l}\right)^{2 s_{l}+1}}\right]_{t=\tau_{\nu}}^{\left(2 s_{\nu}-i-m\right)} \\
& \times \int_{\mathbb{R}}\left(x-\tau_{\nu}\right)^{2 s_{\nu}-m} \prod_{\substack{l=1 \\
l \neq \nu}}^{n}\left(x-\tau_{l}\right)^{2 s_{l}+1} d \lambda(x) .
\end{aligned}
$$

Therefore

$$
A_{i, \nu}=\frac{1}{i !} \sum_{k=0}^{2 s_{v}-i} \frac{1}{\left(2 s_{v}-i-k\right) !}\left[\frac{1}{\omega_{v}(t)}\right]_{t=\tau_{v}}^{\left(2 s_{v}-i-k\right)} \int_{\mathbb{R}}\left(x-\tau_{v}\right)^{2 s_{v}-k} \frac{\prod_{l=1}^{n}\left(x-\tau_{l}\right)^{2 s_{l}+1}}{\left(x-\tau_{v}\right)^{2 s_{v}+1}} d \lambda(x),
$$

that is,

$$
\begin{aligned}
A_{i, \nu}= & \frac{1}{i !\left(2 s_{\nu}-i\right) !} \sum_{k=0}^{2 s_{\nu}-i}\left(\begin{array}{c}
2 s_{\nu}-i \\
k
\end{array}\right)\left[\frac{1}{\omega_{\nu}(t)}\right]_{t=\tau_{\nu}}^{\left(2 s_{\nu}-i-k\right)} \\
& \times \int_{\mathbb{R}} \frac{(-1)^{k+1} k ! \prod_{l=1}^{n}\left(x-\tau_{l}\right)^{2 s_{l}+1}}{\left(\tau_{\nu}-x\right)^{k+1}} d \lambda(x) .
\end{aligned}
$$

For $p=0, \ldots, k, k=0, \ldots, 2 s_{v}-i, i=0, \ldots, 2 s_{v}, v=1, \ldots, n$, we have

$$
\left[\prod_{l=1}^{n}\left(t-\tau_{l}\right)^{2 s_{l}+1}-\prod_{l=1}^{n}\left(x-\tau_{l}\right)^{2 s_{l}+1}\right]_{t=\tau_{v}}^{(p)}= \begin{cases}-\prod_{l=1}^{n}\left(x-\tau_{l}\right)^{2 s_{l}+1} ; & p=0 \\ {\left[\prod_{l=1}^{n}\left(t-\tau_{l}\right)^{2 s_{t}+1}\right]_{l=\tau_{v}}^{(p)}} & ; p>0 .\end{cases}
$$

If $p>0$, then by using the Leibniz formula we have

$$
\begin{aligned}
{\left[\prod_{l=1}^{n}\left(t-\tau_{l}\right)^{2 s_{l}+1}\right]_{t=\tau_{\nu}}^{(p)} } & =\left[\left(t-\tau_{v}\right)^{2 s_{\nu}+1} \omega_{\nu}(t)\right]_{t=\tau_{v}}^{(p)} \\
& =\sum_{m=0}^{p}\left(\begin{array}{l}
p \\
m
\end{array}\right)\left[\left(t-\tau_{\nu}\right)^{2 s_{\nu}+1}\right]_{t=\tau_{v}}^{(m)}\left[\omega_{\nu}(t)\right]_{t=\tau_{v}}^{(p-m)}=0 .
\end{aligned}
$$


Therefore

$$
\left[\prod_{l=1}^{n}\left(t-\tau_{l}\right)^{2 s_{l}+1}-\prod_{l=1}^{n}\left(x-\tau_{l}\right)^{2 s_{l}+1}\right]_{t=\tau_{v}}^{(p)}= \begin{cases}-\prod_{l=1}^{n}\left(x-\tau_{l}\right)^{2 s_{l}+1} ; & p=0 . \\ 0 ; & p>0 .\end{cases}
$$

For the integral in (6.3) we have

$$
\begin{aligned}
\int_{\mathbb{R}} \frac{(-1)^{k+1} k ! \prod_{l=1}^{n}\left(x-\tau_{l}\right)^{2 s_{l}+1}}{\left(\tau_{\nu}-x\right)^{k+1}} d \lambda(x) & \int_{\mathbb{R}} \frac{(-1)^{k} \cdot k !}{\left(\tau_{v}-x\right)^{k+1}}\left(-\prod_{l=1}^{n}\left(x-\tau_{l}\right)^{2 s_{l}+1}\right) d \lambda(x) \\
= & \left(\begin{array}{l}
k \\
0
\end{array}\right) \int_{\mathbb{R}}\left[(t-x)^{-1}\right]_{t=\tau_{v}}^{(k-0)}\left(-\prod_{l=1}^{n}\left(x-\tau_{l}\right)^{2 s_{l}+1}\right) d \lambda(x) \\
& +\sum_{p=1}^{k}\left(\begin{array}{l}
k \\
p
\end{array}\right) \int_{\mathbb{R}}\left[(t-x)^{-1}\right]_{t=\tau_{v}}^{(k-p)}\left[\prod_{l=1}^{n}\left(t-\tau_{l}\right)^{2 s_{l}+1}-\prod_{l=1}^{n}\left(x-\tau_{l}\right)^{2 s_{l}+1}\right]_{t=\tau_{v}}^{(p)} d \lambda(x) \\
= & \sum_{p=0}^{k}\left(\begin{array}{l}
k \\
p
\end{array}\right) \int_{\mathbb{R}}\left[(t-x)^{-1}\right]_{t=\tau_{v}}^{(k-p)}\left[\prod_{l=1}^{n}\left(t-\tau_{l}\right)^{2 s_{l}+1}-\prod_{l=1}^{n}\left(x-\tau_{l}\right)^{2 s_{l}+1}\right]_{t=\tau_{v}}^{(p)} d \lambda(x) \\
= & \int_{\mathbb{R}}\left[\frac{\prod_{l=1}^{n}\left(x-\tau_{l}\right)^{2 s_{l}+1}-\prod_{l=1}^{n}\left(t-\tau_{l}\right)^{2 s_{l}+1}}{x-t}\right]_{t=\tau_{v}}^{(k)} d \lambda(x) .
\end{aligned}
$$

Now (6.3) becomes

$$
\begin{aligned}
A_{i, \nu}= & \frac{1}{i !\left(2 s_{\nu}-i\right) !} \sum_{k=0}^{2 s_{\nu}-i}\left(\begin{array}{c}
2 s_{v}-i \\
k
\end{array}\right)\left[\frac{1}{\omega_{\nu}(t)}\right]_{t=\tau_{\nu}}^{\left(2 s_{\nu}-i-k\right)} \\
& \times \int_{\mathbb{R}}\left[\frac{\prod_{l=1}^{n}\left(x-\tau_{l}\right)^{2 s_{t}+1}-\prod_{l=1}^{n}\left(t-\tau_{l}\right)^{2 s_{l}+1}}{x-t}\right]_{t=\tau_{v}}^{(k)} d \lambda(x),
\end{aligned}
$$

that is, (6.2) holds.

REMARK C. The formula (6.1) has been used for numerical calculation of the coefficients $A_{i, v}$ in (1.4) (see [15]). The expression (6.2) may be of interest for theoretical considerations. For example, the term

$$
\int_{\mathbb{R}} \frac{\prod_{l=1}^{n}\left(x-\tau_{l}\right)^{2 s_{l}+1}-\prod_{l=1}^{n}\left(t-\tau_{l}\right)^{2 s_{l}+1}}{x-t} d \lambda(x)
$$

is similar to the associated polynomials of the second kind (or the numerator polynomials) corresponding to the ordinary orthogonal polynomials (see $[4, \mathrm{p}$. 86]). (In the case of $s_{1}=s_{2}=\cdots=s_{n}=0$ it is precisely that.) 
Acknowledgements. This paper is dedicated to the memory of Professor Alessandro Ossicini. This work was supported in part by the Serbian Ministry of Science and Technology.

\section{References}

[1] I. S. Berezin and N. P. Židkov, Metody Vychislenii. Tom pervyi, Russian (Gosudarstv. Izdat. Fiz.-Mat. Lit., Moscow, 1962).

[2] L. Chakalov, "General quadrature formulae of Gaussian type", Bulgar. Akad. Nauk Izv. Mat. Inst. 1 (1954) 67-84, (Bulgarian); English transl.: East J. Approx. 1 (1995) 261-276.

[3] L. Chakalov, "Formules générales de quadrature mécanique du type de Gauss", Colloq. Math. 5 (1957) 69-73.

[4] T. S. Chihara, An introduction to orthogonal polynomials, Mathematics and its Applications 13 (Gordon and Breach, New York, 1978).

[5] M. Frontini, W. Gautschi and G. V. Milovanović, "Moment-preserving spline approximation on finite intervals", Numer. Math. 50 (1987) 503-518.

[6] M. Frontini and G. V. Milovanović, "Moment preserving spline approximation on finite intervals and Turán quadratures", Facta Univ. Ser. Math. Inform. 4 (1989) 45-56.

[7] W. Gautschi, "Discrete approximations to spherically symmetric distributions", Numer. Math. 44 (1984) 53-60.

[8] W. Gautschi and G. V. Milovanović, " $S$-orthogonality and construction of Gauss-Turán-type quadrature formulae", J. Comput. Appl. Math. 86 (1997) 205-218.

[9] A. Ghizzetti and A. Ossicini, Quadrature formulae (Akademie Verlag, Berlin, 1970).

[10] A. Ghizzetti and A. Ossicini, "Sull" esistenza e unicità delle formule di quadratura gaussiane", Rend. Mat. (6) 8 (1975) 1-15.

[11] G. Golub and J. Kautsky, "Calculation of Gauss quadratures with multiple free and fixed knots", Numer. Math. 41 (1983) 147-163.

[12] G. V. Milovanović, "Construction of $s$-orthogonal polynomials and Turán quadrature formulae", in Numerical Methods and Approximation Theory III (Niš, 1987) (ed. G. V. Milovanović), (Univ. of Niš, Niš, 1988), 311-328.

[13] G. V. Milovanović and M. M. Spalević, "Quadrature formulae connected to $\sigma$-orthogonal polynomials", J. Comput. Appl. Math. (Suppl.) (to appear).

[14] G. V. Milovanović and M. M. Spalević, "A numerical procedure for coefficients in generalized Gauss-Turán quadratures", FILOMAT (formerly Zb. Rad.) 9 (1995) 1-8.

[15] G. V. Milovanović and M. M. Spalević, "Construction of Chakalov-Popoviciu type quadrature formulae", Rend. Circ. Mat. Palermo (Suppl.) (2) II (52) (1998) 625-636.

[16] A. Morelli and I. Verna, "Formula di quadratura in cui compaiono i valori della funzione e delle derivate con ordine massimo variabile da nodo a nodo", Rend. Circ. Mat. Palermo (2) 18 (1969) 91-98.

[17] T. Popoviciu, "Sur une généralisation de la formule d'intégration numérique de Gauss", Acad. $R$. $P$. Romîne Fil. laşi Stud. Cerc. Sti. 6 (1955) 29-57, (Romanian).

[18] M. M. Spalević, "Product of Turán quadratures for cube, simplex, surface of the sphere, $\bar{E}_{n}^{r}, E_{n}^{r^{2}}$ ", J. Comput. Appl. Math. 106 (1999) 99-115.

[19] A. H. Stroud and D. D. Stancu, "Quadrature formulas with multiple Gaussian nodes", J. SIAM Numer. Anal. Ser. B 2 (1965) 129-143.

[20] P. Turán, "On the theory of the mechanical quadrature", Acta Sci. Math. Szeged 12 (1950) 30-37.

[21] G. Vincenti, "On the computation of the coefficients of $s$-orthogonal polynomials", SIAM J. Numer. Anal. 23 (1986) 1290-1294. 